\section{Participación ciudadana en el contexto de la evaluación de tecnologías sanitarias}

\author{
BERENICE FREILE-GUTIÉRREZ ${ }^{\text {la }}$
}

\section{Citizen participation in the context of health technology assessment}

Citizen participation is important in all economic sectors of the democratic world, as it is also in the field of health. This is due to the significant value of life and therefore enjoying this in a healthy condition. This paper seeks to provide an overview of public participation in the context of Health Technology Assessments (HTA), covering from conceptualization to practical experiences. Within the existing literature, the definitions and methodological aspects of the topic discussed are reviewed, as well as how citizen participation in HTA agencies is manifested, presenting barriers as well as enablers for public involvement. In the Chilean case, where there are no public participation experiences in the context of HTA, a review of these initiatives in the health care sector is performed. In light with the studies examined, it is concluded that there is much to advance in the concreteness of citizen participation in HTA's agencies; efforts at institutional level as well as from community organizations are needed. This review can be useful as an antecedent for countries that are considering incorporating HTA in the future.

(Rev Med Chile 2014; 142 (S1): S 27-32)

Key words: Consumer health information; Consumer participation; Patient participation; Technology assessment, biomedical.
${ }^{1}$ Departamento Economía de la Salud. División de Planificación Sanitaria. Subsecretaría de Salud Pública. Ministerio de Salud; Santiago, Chile. angeniero Civil Industrial. Universidad de Chile. Alumna Magister en Salud Pública. Universidad de Chile, beca financiada por CONICYT-PCHA Magíster Nacional/2013-folio 79130005.

Fuente de apoyo financiero: No se recibió apoyo financiero para la escritura del presente artículo. El empleador de la autora (Subsecretaría de Salud Pública) no ha tenido influencia en el diseño ni en el contenido del artículo.

Conflictos de intereses: La autora declara no tener conflictos de intereses. Participa desde agosto 2013 en la Comisión Nacional de Evaluación de Tecnologías Sanitarias.

Este artículo fue sometido a revisión por pares.

Correspondencia a: Berenice Freile Gutiérrez bfreileg@hotmail.com bfreile@minsal.c

\section{Conceptos relativos a la participación ciudadana}

I

a participación ciudadana hace referencia al conjunto de acciones impulsadas por la democracia representativa, mediante la integración de la comunidad al quehacer político. Utiliza mecanismos para que la población acceda a las decisiones estatales, sin formar parte de la administración pública ni de partidos políticos. Según Gadea ${ }^{1}$, la participación engloba cualquier "intervención de los particulares en actividades públicas mediante el ejercicio de sus derechos civiles".

A fines de los 60, Arnstein ${ }^{2}$ definió ocho peldaños para representar la participación ciudadana, comenzando por no participación, que engloba manipulación (parece intervención del público, pero se trata de relaciones públicas conducidas por actores más poderosos) y terapia (la experiencia de un grupo clínico aparece como participación ciudadana). Luego, incluye tres niveles en tokenismo (práctica de hacer pequeñas concesiones, en público, a un grupo minoritario, para desviar acusaciones de prejuicio y discriminación), donde se solicita información, se consulta acerca de materias relacionadas con sintomatologías y se aplaca a la ciudadanía escuchando su visión. Finaliza con tres peldaños de ciudadanía empoderada, que incluye asociación colaborativa del público hacia las instituciones, delegación de poder de dichas instituciones a los participantes, y control de los ciudadanos, que refleja el máximo poder. Este modelo continúa vigente, ya que es citado constantemente por otros autores (Figura 1). 
Figura 1. Peldaños de Participación Ciudadana. Fuente: Arnstein, 1969.

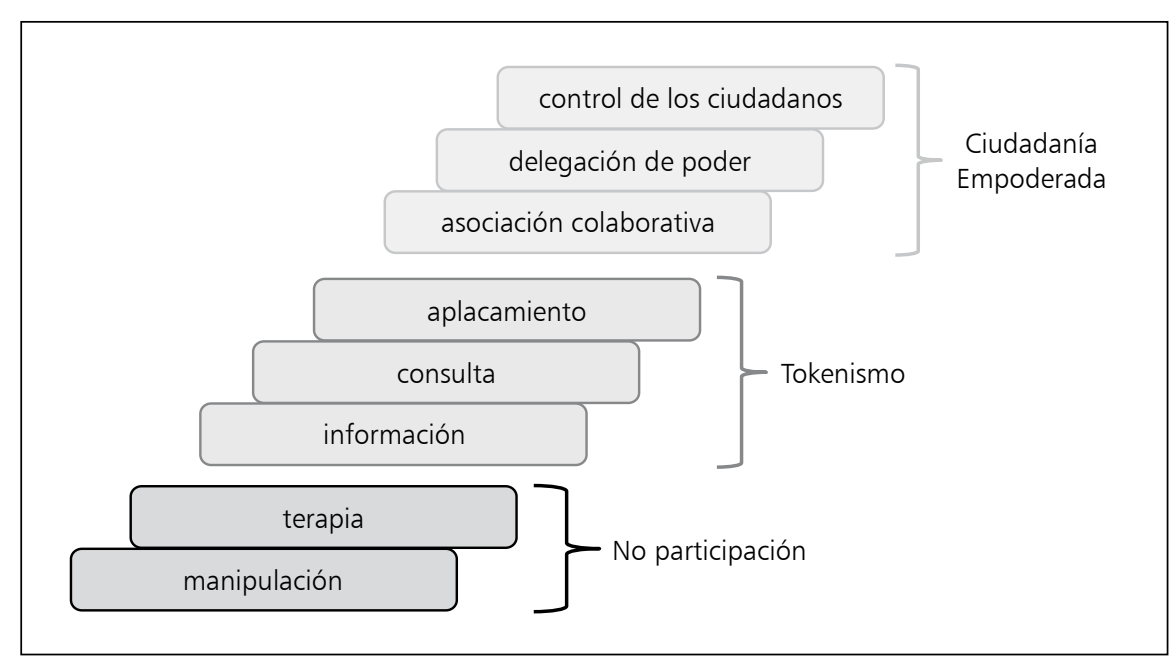

Según Sepúlveda ${ }^{3}$, la participación exitosa necesita de un sector público con apertura, que genere y organice la información, que establezca espacios y mecanismos para acoger las necesidades y propuestas de la ciudadanía. También, precisa de una población que se interese en las cuestiones públicas, mediante organizaciones propias.

En el ámbito de la salud, Segura ${ }^{4}$ definió el concepto de participación ciudadana como un "instrumento orientado a mejorar los resultados de las intervenciones sanitarias". Así, se puede participar en decisiones terapéuticas, ya sea estableciendo prioridades, en la elaboración y evaluación de políticas sanitarias, definiendo y estimando el impacto de intervenciones o servicios de salud a ser ofrecidos, además de la eventual contribución en la selección de intervenciones con financiamiento público.

Se usa en este documento como sinónimos: ciudadano, público, población y consumidor. Este último término, definido por Cochrane Collaboration (2008), como "alguien que usa, es afectado por, o tiene derecho a usar los servicios relativos a salud".

\section{Aspectos que conforman la Participación Ciudadana en Evaluación de Tecnologías Sanitarias}

Según Gauvin et al. ${ }^{5}$, los aspectos constitutivos son: tipos de público, ámbitos de participación y niveles de participación (Figura 2). Los tipos de público son pacientes y/o ciudadanos. Los pacientes incluyen usuarios individuales, sus representantes (padres, hijos, y/o cuidadores) y agrupaciones de enfermos. Los ciudadanos pueden ser individuos, sus representantes, o consumidores organizados.

Los ámbitos de participación son tres: formulación de políticas, aspectos organizacionales e investigación. En la formulación de políticas, el consumidor puede colaborar en definir la cobertura de la política de salud pública, a nivel de planes de seguro, como los servicios de salud ofrecidos. En el ámbito organizacional, el aporte del público se orienta hacia la forma de dirección de la agencia de Evaluación de Tecnologías Sanitarias (ETESA). En investigación, el ciudadano puede participar en definición de preguntas de investigación, identificación de resultados (outcomes), recolección y evaluación de evidencia, así como escritura y revisión de informes.

Por último, existen tres niveles de participación ciudadana: información, consulta y participación. La información se refiere a difusión y comunicación con el público. La consulta considera diferentes métodos de detección de necesidades y/o preferencias de los consumidores (encuestas, modelos de elección discreta, focus groups, ejercicios de disposición a pagar, entrevistas en profundidad, entre otros). El nivel de participación, más relacionado con el concepto de "ciudadanía empoderada" de Arnstein ${ }^{2}$, comprende la colaboración de pacientes y/o público en los procesos ETESA, su compromiso, y cuando es posible, su control sobre dichos procesos. El consumidor puede par- 


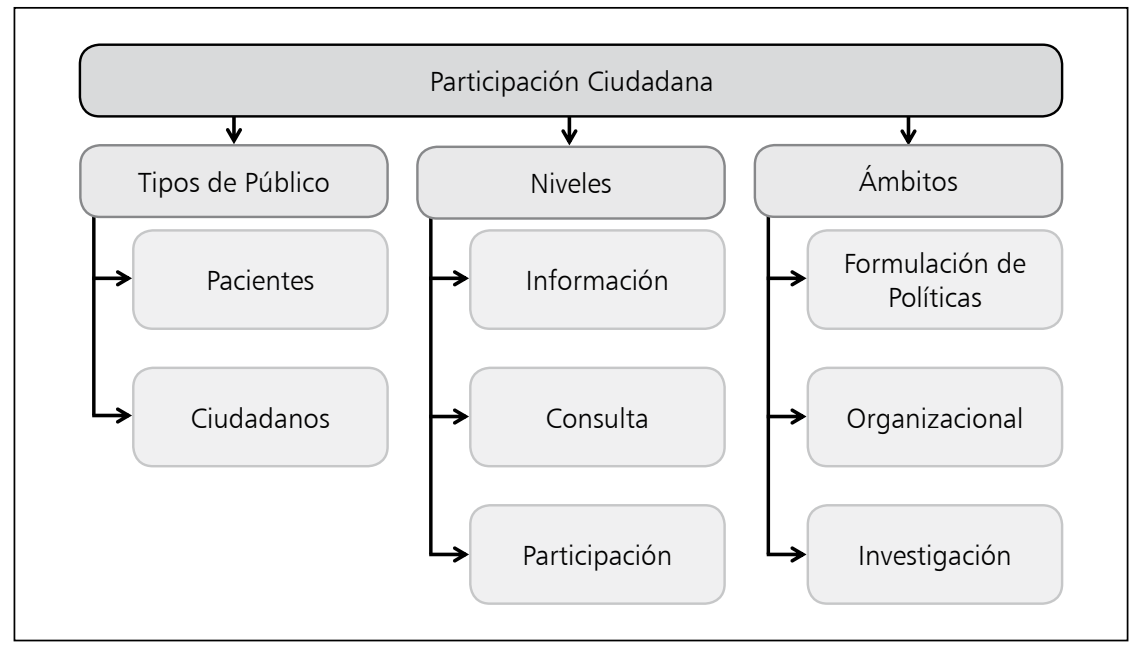

Figura 2. Aspectos de la participación del público en ETESA. Fuente: Gauvin, 2010. ticipar en priorización de tecnologías, definición del alcance (scoping), evaluación de evidencia, y difusión de resultados. Su intervención puede incluir desde la generación de comentarios en propuestas de investigación, respuestas a preguntas específicas, revisión de documentos en borrador, hasta la apelación o reclamo a la recomendación hecha por la agencia.

\section{Métodos de participación en ETESA}

Según Abelson et al. ${ }^{6}$, se utilizan métodos deliberativos y no deliberativos en la participación ciudadana en ETESA. La diferencia entre los métodos está dada por el término "deliberación", definido como: "análisis y ponderación de los factores implicados en el tema, para disminuir su incertidumbre"?.

Abelson et al. ${ }^{6}$ distinguen doce métodos no deliberativos: paneles de ciudadanos, focus groups, ejercicio de creación de consenso, encuestas, audiencias públicas, puertas abiertas, comité consultivo ciudadano, planificación comunitaria, visión, notificación, distribución y solicitud de comentarios, referéndum y referéndum de valor estructurado. También diferencian cinco métodos deliberativos: jurado de ciudadanos, consejo de ciudadanos, grupos de planificación, conferencia consensuada y votación deliberativa.

Todos los métodos señalados requieren, para tener una operación exitosa, de ciudadanos representativos de la población o comunidad que encarnan, lo que no siempre es posible. Los métodos no deliberativos tienen una participación más estructurada, de menor costo y más centrada en consultas hacia la ciudadanía. En contraste, los métodos deliberativos son de mayor costo, promueven la discusión y el consenso, son más intensivos en tiempo del consumidor y de los organizadores, $y$ el tema a tratar se establece $a$ priori, con entrega anticipada de información concerniente a dicho tema. Cada método tiene una forma de operación característica, así como sus fortalezas y debilidades.

\section{Experiencias de Participación Ciudadana en ETESA}

En la encuesta de Hailey y Nordwall ${ }^{8}$, aplicada a las agencias pertenecientes a International Network of Agencies for Health Technology Assessment (INAHTA), destacan los siguientes resultados: Existe participación de los consumidores en algunos aspectos de los procesos de ETESA, aunque no sistemáticamente (57\%); gran número de agencias cuentan con resúmenes de informes de ETESA adecuados para los ciudadanos (49\%); parte de los consumidores participan en la difusión de material de ETESA (36\%); y la mayor parte de las agencias están interesadas en involucrar al público en el futuro (83\%).

Para América Latina y el Caribe, Arellano et al. ${ }^{9}$ destacan que no hay difusión a pacientes y consumidores. La difusión de la información (uno de los objetivos de INAHTA), apunta a terceros 
pagadores $(55 \%)$ y creadores de políticas del gobierno (41\%).

Por otra parte, la modelación de los 15 principios claves para una mejor conducción de ETESA, planteados por Drummond et al. ${ }^{10}$, verifican que algunas organizaciones involucran a los interesados en diversas etapas del proceso; en la determinación del alcance de la evaluación, en comentarios al informe preliminar, y en audiencias. Estas agencias son: National Institute for Health and Clinical Excellence (NICE-Reino Unido), Institute for Quality and Efficiency in Health Care (IQWiGAlemania) y Agency for Healthcare Research and Quality (AHRQ-Estados Unidos).

El estudio de Neumann et al. ${ }^{11}$ revisa la adherencia de las agencias a los principios clave $^{10}$. Se muestra los resultados para los principios 10 (involucramiento activo de todos los actores relevantes) y 14 (comunicación adecuada a los distintos tomadores de decisiones), atingentes a participación ciudadana. Así, las agencias que mejor adhieren al principio 10 son cinco: Centers for Medicare and Medicaid Services (CMS-Estados Unidos), NICE, IQWiG, Dental and Pharmaceutical Benefits Agency (TLV-Suecia) y Canadian Agency for Drugs and Technologies in Health (CADTH). Las Agencias que mejor cumplen el principio 14 son cinco: NICE, IQWIG, Swedish Council on Health Technology Assessment (SBU), CADTH y German Agency for Health Technology Assessment at the Institute for Medical Documentation and Information (DAHTA@DIMDI).

El mismo estudio ${ }^{11}$ indica que, las agencias consideradas están en diferentes etapas de desarrollo, como Pharmaceutical Benefits Advisory Committee (PBAC-Australia) y NICE, que están bien consolidadas, mientras que otras, como Health Insurance Review Agency (HIRA-Corea del Sur), están recién surgiendo.

Para América Latina y el Caribe, de acuerdo a la encuesta de Pichon-Riviere et al. ${ }^{12}$, aplicada a investigadores de ETESA (14\%) y a usuarios (86\%), de Argentina, Perú, Brasil, Colombia, Uruguay, México, Chile y El Caribe, existe una gran brecha entre la relevancia que tienen los principios para los encuestados y su grado de aplicación. Además, la comunicación adecuada hacia los distintos tomadores de decisiones está muy lejos del ideal.

A objeto de mejorar la comparación entre agencias, Drummond et al. ${ }^{13}$ definieron preguntas para cada principio. Algunas preguntas destacables para el principio 10 son: ¿la agencia de ETESA exige formalmente el compromiso de participación de las partes interesadas en sus actividades?, ¿las involucra en la definición del alcance de las evaluaciones?, ¿pueden comentar los informes borrador? , y ison incluidos en los comités de actores relevantes (grupos de pacientes, fabricantes de tecnología o especialistas clínicos, según corresponda)?. Algunas preguntas para el principio 14 son: ¿tiene la agencia plan de comunicaciones para sus recomendaciones y decisiones?, ¿producen distintas versiones de informes para diferentes audiencias(profesionales de la salud, tomadores de decisiones y/o público general)?, y ¿se monitorea y evalúa la efectividad de la comunicación?.

Por último, la revisión sistemática reciente de Gagnon et al. ${ }^{14}$ relata prácticas exitosas en 24 estudios, correspondientes a 8 países investigados. Existe involucramiento del público en dos niveles: consulta y participación (el nivel información no es abordado en el estudio). También se desarrolla participación en todos los ámbitos: organizacional, formulación de políticas e investigación, utilizando métodos deliberativos y no deliberativos. Los aspectos considerados son muy diversos, desde la consulta por disposición a pagar por una nueva tecnología, la discusión de uso para un determinado medicamento y su reembolso, la generación de los criterios de priorización de las evaluaciones, hasta la definición de la participación del público en temas complejos.

\section{Principales Barreras y Facilitadores para la Participación Ciudadana en ETESA}

Según Gagnon et al. $^{14}$, identifican entre las barreras: la forma de reclutamiento de los ciudadanos (por ser un tema delicado y buscar representatividad, además que las agencias de ETESA prefieren a grupos organizados e informados que a ciudadanos, pacientes y usuarios individuales); la velocidad de las discusiones, el lenguaje técnico y la escasa familiarización con los procesos de ETESA; el tiempo y otros recursos adicionales requeridos; el interés de algunos actores relevantes en mantener el statu quo; y el momento de integración del público (si así sucede, normalmente es en etapas posteriores al término del informe de ETESA). No se reporta evidencia acerca de la participación ciudadana en la toma de decisiones en ETESA.

Para Latinoamérica y El Caribe, algunas barre- 
ras de involucramiento ciudadano planteadas por Celedón y Noé ${ }^{15}$ son: la desigualdad en la distribución de ingresos y en otras formas de poder; el escaso e incipiente conocimiento de los derechos del consumidor frente a los servicios públicos y los mínimos mecanismos que permitan hacerlos efectivos; la precariedad para reconocer la diversidad social y recoger la opinión de las minorías y/o de sectores más marginados; y el débil desarrollo de las organizaciones sociales. Por ello, en general, las políticas públicas, incluidas las políticas de participación, se construyen desde el Estado, más que por iniciativa de los ciudadanos.

Por otra parte, Gagnon et al. ${ }^{14}$ identifican facilitadores para lograr una mejor participación ciudadana en ETESA, las cuales incluyen: entrenamiento, soporte y entrega previa de información útil; invitación a ciudadanos con experiencia en el tema a tratar; ajuste de los tiempos para cada tipo de actividad; $y$ transparencia de procesos con rendición de cuentas de las agencias. Finalmente, Bridges y Jones ${ }^{16}$ puntualizan que en el futuro es importante focalizar los intereses, ya sea del gobierno, la industria, la academia, o la medicina, en " $e l$ bienestar del paciente", junto con "empoderar" a los pacientes (tomar decisiones informadas respecto de su propia salud), dentro de los procesos de ETESA.

\section{Experiencias en Chile de Participación Ciudadana en Salud}

Existe en Chile un Instructivo Presidencial de Participación Ciudadana $(2001)^{3}$, que adhiere a las concepciones ya revisadas. Se presentan algunos casos de participación pública en salud.

La Estrategia Nacional de Salud 2011-2020 del Ministerio de Salud (MINSAL) ${ }^{17}$, plantea en el punto 7.6: "Aumentar a 100\% la proporción de Servicios de Salud y Secretarías Regionales Ministeriales de Salud que han implementado mecanismos de participación ciudadana y usuaria, y ejercicio de derechos". El diagnóstico (2009) indicó que: todos los Servicios de Salud (SS) cuentan con instancias de participación social; 8 de los 29 SS tienen representantes de los usuarios en el Consejo Integrador de la Red Asistencial (CIRA); 29 SS y 133 hospitales poseen cuentas públicas participativas; y $25 \%$ de los SS y $10 \%$ de los hospitales han establecido compromisos explícitos con la comunidad. Mediante consejos de la sociedad civil (consultivos o de desarrollo), comités locales de salud y otros, se busca mejorar la incidencia ciudadana en la gestión pública de salud.

Asimismo, el Organismo Andino de SaludConvenio Hipólito Unanue (ORAS-CONHU) creó un consejo consultivo (2009) para prevención del embarazo adolescente, donde participan dos jóvenes (mujer y hombre) elegidos en cada región de Chile.

Igualmente, en el Proceso de Garantías Explícitas de Salud (GES) ${ }^{18}$, se aplicó focus groups (2008) para conocer la importancia asignada por la población a los problemas de salud incluidos en las garantías (método no deliberativo, ámbito formulación de política, nivel consulta). El Consejo consultivo GES presentó en Antofagasta (2006) el Decreto 170 (25 patologías GES), incluyendo testimonios de pacientes y diálogo ampliado con la sociedad civil (método no deliberativo, ámbito formulación de politica, nivel información).

Por último, MINSAL realizó tres estudios de Preferencias Sociales GES ${ }^{19}$ : i) Priorizaciones de inversiones en salud (1996), primera investigación cualitativa para conocer y priorizar las necesidades en salud desde el punto de vista de los usuarios del sistema; ii) Estudios iniciales de percepción de salud (2000), proceso consensuado de formulación de políticas de salud integrales con participación de los usuarios y otros actores sociales relevantes, además de la elaboración de propuestas de rediseño del sector salud: público y privado; iii) Determinación de prioridades en salud propuestas en la implementación de las GES (2005), estudio que ordenó el listado de 31 problemas de salud a ser implementados en el marco legal de las GES, en base a criterios para definir prioridades de atención en salud de la población.

\section{Conclusiones}

El presente documento provee una visión general de la participación ciudadana en el contexto de ETESA, revisando tanto los conceptos, como las experiencias prácticas que reporta la literatura. Se ilustra además, el caso chileno de participación de los ciudadanos en salud.

Aunque se considera importante la participación del público, pocas organizaciones y/o agencias de ETESA tienen métodos estructurados para dicha participación en investigaciones y/o en los procesos de ETESA. Se requiere refinar los mecanismos orientados a mejorar la participación 
ciudadana, así como para medirla y compararla entre las distintas agencias.

Además, la transparencia y la promoción en participación del consumidor, efectuados por las agencias ETESA y/o por el Estado, propenderían al empoderamiento decisional de la población respecto de la propia salud. Falta también mejorar las organizaciones sociales, para lograr una mejor participación en este ámbito.

Por último, falta consensuar, entre las agencias y las organizaciones sociales, el momento óptimo de inclusión de la ciudadanía, tanto en la investigación como en los procesos ETESA, aquel en que se obtendría mayores beneficios en el bienestar del paciente.

Todo lo anterior, puede servir de base para el diseño de ETESA en los países que las incorporen en el futuro.

\section{Referencias}

1. Gadea ME. Las políticas de participación ciudadana. Nuevas formas de relación entre la administración pública y la ciudadanía. El caso de la ciudad de Valencia y su área metropolitana. Tesis doctoral. Valencia: Servei de Publicacions de la Universitat de Valencia 2007. 97. Disponible en: [http://www.tdx.cat/bitstream/handee/10803/10296/gadea.pdf;jsessionid=E73772C0119CA 5D83C84318CCB50DFA9.tdx2?sequence=1.

2. Arnstein S. A ladder of citizen participation. JAIP 1969. 35: 216-24.

3. Sepúlveda J. ¿Qué es la Participación Ciudadana? 2004, UC Temuco. Disponible en: [ http://www.trabajosocial. uct.cl/wp-content/uploads/aproximacion-al-conceptode-participacion-ciudadana.

4. Segura A. La participación ciudadana, la sanidad y la salud. Elementos para un debate informado. GCS 2010. $12 ; 2$.

5. Gauvin F, Abelson J, Giacomini M, Eyles J, Lavis JN. "It all depends": conceptualizing public involvement in the context of health technology assessment agencies. Soc Sci Med 2010; 70 (10): 1518-26.

6. Abelson J, Forest P-G, Eyles J, Smith P, Martin E, Gauvin F-P. A Review of Public Participation and Consultation Methods. CHEPA. Working Paper 01-04, 200. Disponible en: [http://www.citizenshandbook.org/compareparticipation.pdf.

7. FELAIBE. Federación Latinoamericana y del Caribe de Bioética y Fundación Ciencia y Vida. Ética Clínica y Comités de Ética en Latinoamérica 2011, 156. Disponible en: [http://medicina.udd.cl/centro-bioetica/
files/2011/09/FELAIBE-Comites-deliberacion.pdf

8. Hailey D, Nordwall M. Survey on the involvement of consumers in health technology assessment programs. Int J Technol Assess Health Care 2006; 22 (4): 497-9.

9. Arellano L, Reza M, Blasco JA, Andradas E. A content analysis of Health Technology Assessment programs in Latin America. Int J Technol Assess Health Care 2009. 25 (4): 570-6.

10. Drummond M, Schwartz JS, Jonsson B, Luce BR, Neumann PJ, Siebert U, et al. Key principles for the improved conduct of health technology assessments for resource allocation decisions. Int J Technol Assess Health Care 2008; 24 (3): 244-58; discussion 362-8.

11. International Working Group for HTA. Are Key Principles for improved health technology assessment supported and used by health technology assessment organizations? Int J Technol Assess Health Care 2010; 26 (1): 71-8.

12. Pichon-Riviere A, Augustovski F, Rubinstein A, Marti SG, Sullivan SD, Drummond MF. Health technology assessment for resource allocation decisions: are key principles relevant for Latin America? Int J Technol Assess Health Care 2010; 26 (4): 421-7.

13. Drummond M, Neumann P, Jonsson B, Luce B, Schwartz JS, Siebert U et al. Can we reliably benchmark health technology assessment organizations? Int J Technol Assess Health Care 2012; 28 (2): 159-65.

14. Gagnon M, Desmartis M, Lepage-Savary D, Gagnon J, StPierre M, Rhainds $M$, et al. Introducing patients' and the public's perspectives to health technology assessment: A systematic review of international experiences. Int J Technol Assess Health Care 2011; 27 (1):31-42.

15. Celedón C, Noé M. Reformas del sector de la salud y participación social. Rev Panam Salud Publica/Pan Am J Public Health 8 (1/2) 2000.

16. Bridges J, Jones C. Patient-based health technology assessment: a vision of the future. Int J Technol Assess Health Care 2007; 23 (1): 30-5.

17. MINSAL 2011. Ministerio de Salud. Estrategia Nacional de Salud para el cumplimiento de los Objetivos Sanitarios de la Década 2011-2020. Disponible en: [http:/] www.minsal.gob.cl/portal/url/item/c4034eddbc96ca6de0400101640159b8.pdf].

18. MINSAL 2012. Ministerio de Salud. Revisión del Proceso de Priorización de las Garantías Explicitas en Salud (GES). Disponible en: [http://desal.minsal.cl/].

19. MINSAL 2008. Ministerio de Salud. Estudio de Preferencias Sociales para la definición de Garantías Explícitas en Salud. INFORME FINAL. Disponible en: [http://epi. minsal.cl/epi/html/invest/IF_ESTUDIO_PREFERENCIAS_SOCIALES_GES_2009.pdf]. 